\title{
Branching on a Sierpinski graph
}

\author{
S. Leorato ${ }^{\mathrm{a}, *}$, E. Orsingher ${ }^{\mathrm{b}}$ \\ a Dipartimento di Studi Economico-Finanziari e Metodi Quantitativi, University of Rome Tor Vergata, via Columbia 2, 00133 Rome, Italy \\ ${ }^{\mathrm{b}}$ Dipartimento di Statistica, Probabilità e Statistiche Applicate, University of Rome La Sapienza, p.le A. Moro 5, 00185 Rome, Italy
}

\section{A R T I C L E I N F O}

\section{Article history:}

Received 10 July 2007

Received in revised form 26 March 2008

Accepted 23 July 2008

Available online $\mathrm{xxxx}$

\section{MSC:}

$60 \mathrm{G} 18$

$60 \mathrm{~J} 80$

\begin{abstract}
A B S T R A C T
The descending motion of particles in a Sierpinski gasket subject to a branching process is examined. The splitting on escape nodes of falling particles makes the event of reaching the base of the gasket possible with positive probability. The r.v.'s $Y(k)$, representing the number of particles reaching level $k$ (that is the $k$-th generation) is the main object of our analysis. The transition probabilities, the means and variances of $Y(k)$ are obtained explicitly with a number of recursive formulas concerning the probability generating functions $\mathbb{E} t^{Y(k)}, k \geq 1$. A section is also devoted to the analysis of extinction probabilities for the branching process developing in this specific fractal set.
\end{abstract}

(C) 2008 Published by Elsevier B.V.

\section{Introduction and description of the process}

Random motions combined with branching have been studied in the context of diffusions and have also originated the theory of superprocesses (McKean, 1975; Bramson, 1978).

Branching processes for particles moving at finite velocity have been examined in some papers appearing in the physical and probabilistic literature (see Ratanov (2006) and references therein). In both cases the processes develop in Euclidean spaces. Diffusions on planar fractals have been studied since the Eighties by physicists and probabilists (see Barlow and Perkins (1988), Barlow (1998) and Dafydd Jones (1996)). The structures where these diffusions are inserted are the Sierpinski gaskets and carpets or some of their generalizations (Kumagai, 1993; Metz, 1993; Barlow and Bass, 1989). The Sierpinski gasket is perhaps the simplest mathematically tractable form of fractal structure and for this reason has maintained a central role throughout the literature, including the most recent one (Teufl, 2003; Osada, 2007).

In our previous paper (2007), we have studied the downward motion under the action of gravity of a single particle on a vertical Sierpinski gasket. In our case, the particle cannot repeatedly visit the same point and this makes our model significantly different from those leading to diffusion. In the present paper, the downward motion on the Sierpinski gasket is combined with a branching process. Even in the pioneering papers it is remarked that the diffusing particle (on carpets or gaskets) barely leaves the starting point. The same type of behaviour has been noticed in the downward motion studied by Leorato and Orsingher (2007). The branching process introduced here contrasts the disintegration of the moving particles (which can undergo absorption at each step). Instead of branching, we supposed that a growing number of particles was poured on the starting point (the upper vertex of the Sierpinski gasket) in order to make the attainment of the base possible. We here assume that on the vertex $V$ (corresponding to level 0 ) of a Sierpinski pregasket $G_{n}$, a particle begins a descending motion with the following rules.

The pregasket $G_{n}$ possesses $2^{n}$ knots (called also nodes) which can be classified as absorbing or escape points (from which further descent is possible). Each particle lying on escape knots at level $k$ splits into two particles (moving independently)

\footnotetext{
* Corresponding author.

E-mail addresses: samantha.leorato@uniroma2.it (S. Leorato), enzo.orsingher@uniroma1.it (E. Orsingher).
} 


\section{ARTICLE IN PRESS}

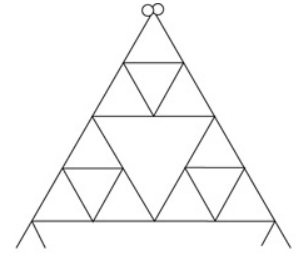

Duplication at level $k=0$

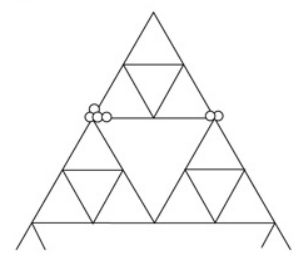

Duplication

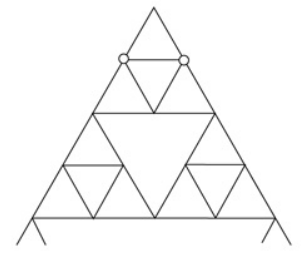

$k=1 Y(1)=2$

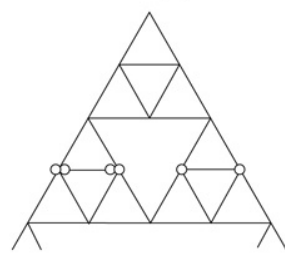

$k=3 Y(3)=6$

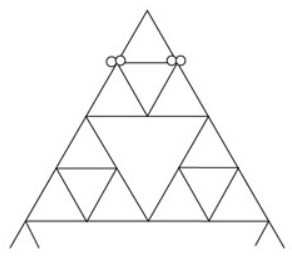

Duplication

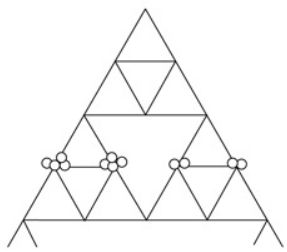

Duplication

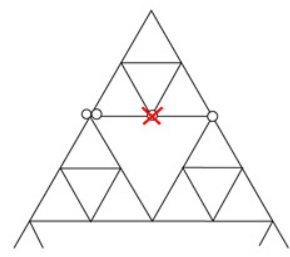

$k=2 Y(2)=3$

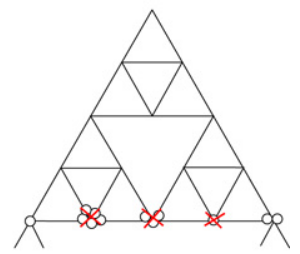

$k=4 Y(4)=3$

Fig. 1. A realization of the process $Y(k)$ after the occurrence of the first duplication. The symbols " $X$ " refer to particles fallen on absorbing states.

which can reach the two neighbouring nodes of level $k+1$ with equal probability $1 / 2$. If an offspring falls on an absorbing knot, it is absorbed and dies.

Particles of the $k$ th generation are those located at level $k$. Thus the particle located at the vertex $V$ represents the initial generation and the two offspring lying at level 1 represent the first generation. The number $Y(k)$ of components of the successive generations $(k \geq 2)$ is random and the birth and death rates depend on the number of escape nodes encountered during the descent.

The principal aim of this paper is the analysis of the distributions and properties of the process $Y(k), k \geq 0$. In particular, we obtain the transition probabilities $\operatorname{Pr}\{Y(k)=n \mid Y(k-1)=m\}$ for all values of $k, n$ and $m$, the mean value $\mathbb{E} Y(k)$ and the explicit expression of $\operatorname{Var} Y(k)$. Furthermore, recursive relationships for the factorial moments are derived and the last section is devoted to the analysis of extinction probabilities. Also the martingale behaviour of the subsequence $Y\left(2^{n}\right)$ is examined.

The original idea which motivated our first paper was to model the falling movement of liquids in porous materials where the internal structure of the matter is idealized as a disordered ensemble of intersecting filaments. We here imagine that the fractal structure causes the splitting of the falling drops, thus igniting the branching process.

\section{Transition probabilities}

We begin by describing the descending motion and the associated branching process. We assume that at the vertex $V$ (level 0 ) a single particle is located and immediately splits into two offspring, both of which step down to level 1, moving independently. Since both nodes of the first level are escape nodes, each of the two offspring will continue its descent. This means that they are not absorbed (they "survive") with probability 1 (i.e. $\operatorname{Pr}\{Y(1 \hat{)}=2\}=1$ ). Instead, each of their 4 offspring can fall with probability $1 / 2$ onto an absorbing node (and therefore die) and thus $Y(2)$ ranges from 0 to 4 (see Fig. 1). It is easy to see that each particle belonging to an even generation, produces two surviving offspring with probability 1. This means that, if $Y(2 k)=i$, then $Y(2 k+1)=2 i$. The transition from odd to even levels is mûch more complicated.

The number of escape knots at level $k$ of the pregasket $G_{n}$ (denoted by $\left.v_{n}(k)\right)$ satisfies the following recursive relationships

$$
v_{n+1}(k)=\left\{\begin{array}{ll}
v_{n}(k) & 0 \leq k \leq 2^{n}-1 \\
2 v_{n}\left(k-2^{n}\right) & 2^{n} \leq k \leq 2^{n+1}-1
\end{array} \quad v_{n}(0)=v(0)=1=2^{0} .\right.
$$

The number $n$ represents the order of fragmentation of $G_{n}$, which possesses $2^{n}+1$ levels (including the vertex and the base).

The autosimilarity explains why $v_{n+1}(k)=v_{n}(k)$ for $0 \leq k<2^{n}$ (the pregasket $G_{n}$ coincides with the upper half of $G_{n+1}$ ) while the lower part of the pre-gasket $G_{n+1}$ is a double copy of $G_{n}$ and this implies that $v_{n+1}(k)=2 v_{n}\left(k-2^{n}\right)$.

Remark 2.1. The function $v_{n}$ can be expressed in terms of the function $\beta_{n}$ introduced in Leorato and Orsingher (2007) as follows:

$$
v_{n}(k)=2^{\beta_{n}(k)}, \quad 0 \leq k \leq 2^{n}-1, n=0,1, \ldots
$$

The function $\beta_{n}(k)$ is defined by the recursive relationship

$$
\beta_{n+1}(k)= \begin{cases}\beta_{n}(k) & 0 \leq k \leq 2^{n}-1 \\ \beta_{n}\left(k-2^{n}\right)+1 & 2^{n} \leq k \leq 2^{n+1}-1\end{cases}
$$


with $\beta_{n}(0)=0, n=0,1,2 \ldots$ From (2.3), with $k=1$, we get that $\beta_{n}(1)=1$, for all $n=1,2, \ldots$ (namely, because the two levels of the first node are escape nodes).

We first note that $v_{n}(0)=2^{0}=2^{\beta_{n}(0)}$, for all $n$. Then, the relationship (2.2) can be easily proved by induction.

From the definition of $v_{n}$ (or of $\beta_{n}$ ), one can easily see that $v_{n}\left(2^{m}\right)=2$ for all $m<n$ and $v_{n}\left(2^{m}-1\right)=2^{m}$ for all $m \leq n$. In fact, from (2.1) we have that

$$
v_{n}\left(2^{m}\right)=v_{n-1}\left(2^{m}\right)=\cdots=v_{m+1}\left(2^{m}\right)=2 v_{m}\left(2^{m}-2^{m}\right)=2 \cdot v_{m}(0)=2
$$

and also that

$$
\begin{aligned}
v_{n}\left(2^{m}-1\right) & =\cdots=v_{m}\left(2^{m}-1\right)=2 v_{m-1}\left(2^{m}-1-2^{m-1}\right)=2 v_{m-1}\left(2^{m-1}-1\right) \\
& =2^{2} v_{m-2}\left(2^{m-2}-1\right)=\cdots=2^{m-1} v_{1}(1)=2^{m} .
\end{aligned}
$$

We observe that the functions $\beta_{n}$ and $v_{n}$ are constant in $n$ within their support. In other words, $\beta_{n}(k)=\beta_{m}(k)$ (resp. $\left.v_{n}(k)=v_{m}(k)\right)$ for all $k$ for which both functions are defined, i.e. for all $k \leq 2^{\min \{n, m\}}-1$. Therefore, in the following we shall omit the subscript $n$ in $v_{n}$.

Some further useful properties of the function $v(k), k \geq 0$ are presented in the following lemmas.

Lemma 2.1. For all $k \geq 0$,

$$
v(k)=2^{p}
$$

where $p$ is the number of ones in the binary representation of $k$.

Proof. By definition $v(0)=1$ and $v(1)=2$ while by drawing a picture, it is evident that $v(2)=2$.

In view of (2.1) for all $2^{m_{1}-1} \leq k \leq 2^{m_{1}}-1$ we can write that:

$$
v(k)=2 v\left(k-2^{m_{1}-1}\right) .
$$

If $k-2^{m_{1}-1}=0$ we have that $v(k)=2 v(0)=2$, otherwise we must continue the procedure above.

Thus, if $k-2^{m_{1}-1} \geq 1$, there exists an integer $1 \leq m_{2}<m_{1}$, such that $2^{m_{2}-1} \leq k-2^{m_{1}-1} \leq 2^{m_{2}}-1$. By applying (2.1) once again, we find that

$$
v\left(k-2^{m_{1}-1}\right)=2 v\left(k-2^{m_{1}-1}-2^{m_{2}-1}\right) .
$$

By combining (2.6) and (2.7) we have that

$$
v(k)=2^{2} v\left(k-2^{m_{1}-1}-2^{m_{2}-1}\right) .
$$

If $k-2^{m_{1}-1}-2^{m_{2}-1}=0$ we have done, otherwise, we continue our procedure in the same manner, until for some integer $p$ we have that

$$
k-2^{m_{1}-1}-\cdots 2^{m_{p}-1}=0
$$

and

$$
v(k)=2^{p} v\left(k-2^{m_{1}-1}-\cdots-2^{m_{p}-1}\right)=2^{p} v(0)=2^{p}
$$

which proves the claim.

Remark 2.2. From Lemma 2.1, we can easily derive some properties of $v(k), k \geq 0$.

(i) An immediate consequence of (2.5) is that, for all $m \geq 0, v\left(2^{m}\right)=2$ and $v\left(2^{m}-1\right)=2^{m}$, as was already proved in Remark 2.1.

(ii) For all $k \geq 0, v(k)=v(2 k)$. This follows straightforwardly from the fact that, if $x_{1} x_{2} x_{3} \cdots x_{m}$ is the binary representation of $k$, with $x_{i}=\{0,1\}, i=1, \ldots, m$, then the sequence $x_{1} x_{2} x_{3} \cdots x_{m} 0$ is the binary representation of $2 k$.

(iii) Moreover, $v(2 k)-v(2 k-1) \leq 0$, for all $k \geq 1$. In order to check this statement, we remark that for every number $k$ there is a number $q_{k}$ such that the rightmost subsequence of length $q_{k}+1$ of the binary representation of $k$ - corresponding to the first $q_{k}+1$ powers of 2 - is composed by a 1 and $q_{k}$ consecutive zeros. For example, for $k=40$, we have the binary representation 101000 and $q_{40}=3$. In particular, $q_{k}=0$ if and only if $k$ is odd, otherwise $q_{k} \geq 1$.

Now, the binary representations of the two numbers $2 k$ and $2 k-1$ differ only for the last $q_{k}+1$ numbers, where ones and zeros are interchanged. For example, if $2 k=40$, the binary representation of $2 k-1=39$ is in fact 100111 . Thus, if $p_{2 k}$ is the total number of ones in the binary representation of $2 k$, we have that $v(2 k)=2^{p_{2 k}} \leq 2^{p_{2 k}+q_{2 k}-1}=2^{p_{2 k-1}}=v(2 k-1)$.

We are now interested in calculating, for an arbitrary pregasket $G_{m}$ (with $2^{m}$ levels), how many layers possess $2^{j}$ escape knots (from each escape knot two descending branches originate). In symbols, we want to evaluate the number of elements belonging to the sets:

$$
\mathcal{A}_{m, j}=\left\{0 \leq k \leq 2^{m}-1: v(k)=2^{j}\right\}, \quad m \geq 1, j=0, \ldots, m .
$$


Lemma 2.2. For all $m \geq 1,0 \leq j \leq m$,

$$
\#\left\{\mathcal{A}_{m, j}\right\}=\left(\begin{array}{c}
m \\
j
\end{array}\right) .
$$

Proof. We proceed by induction and note that for $m=1$ we have two possible levels, the first one with a single escape knot (i.e. $v(0)=1)$ and the other one with two escape knots $(v(1)=2$ ) and thus (2.8) holds.

We assume now that (2.8) is valid for some $m \geq 1$ and prove that it also holds for $m+1$.

$$
\begin{aligned}
\#\left\{\mathcal{A}_{m+1, j}\right\} & =\#\left\{\mathcal{A}_{m, j}\right\}+\#\left\{2^{m} \leq k \leq 2^{m+1}-1: v(k)=2^{j}\right\} \\
& \stackrel{(\text { by }(2.1))}{=} \#\left\{\mathcal{A}_{m, j}\right\}+\#\left\{2^{m} \leq k \leq 2^{m+1}-1: 2 v\left(k-2^{m}\right)=2^{j}\right\} \\
& =\left(\begin{array}{c}
m \\
j
\end{array}\right)+\#\left\{0 \leq k \leq 2^{m}-1: v(k)=2^{j-1}\right\} \\
& =\left(\begin{array}{c}
m \\
j
\end{array}\right)+\#\left\{\mathcal{A}_{m, j-1}\right\}=\left(\begin{array}{c}
m \\
j
\end{array}\right)+\left(\begin{array}{c}
m \\
j-1
\end{array}\right)=\left(\begin{array}{c}
m+1 \\
j
\end{array}\right) .
\end{aligned}
$$

Remark 2.3. We have the following straightforward consequences of the lemma above.

$$
\sum_{k=0}^{2^{m}-1} v(k)=\sum_{j=0}^{m} 2^{j}\left(\begin{array}{c}
m \\
j
\end{array}\right)=3^{m} \text { and } \sum_{k=0}^{2^{m}-1} \frac{1}{v(k)}=\sum_{j=0}^{m} 2^{-j}\left(\begin{array}{c}
m \\
j
\end{array}\right)=\left(\frac{3}{2}\right)^{m} .
$$

Let us introduce the following probabilities

$$
\begin{aligned}
\rho(k) & =\operatorname{Pr}\left\{\begin{array}{l}
\text { a particle at level } k \text { moves to an } \\
\text { escape knot at level } k+1
\end{array}\right\} \\
& =\frac{\#\{\text { directions from level } k \text { to level } k+1\}}{\# \text { dire knots of level } k+1\}}=\frac{v(k+1)}{2 v(k)} .
\end{aligned}
$$

The number $2 v(k)$ in the denominator of (2.10) corresponds to the fact that from each of the $v(k)$ knots at level $k$, two branches descend to level $k+1$.

In view of (2.10), we are able to give the transition probabilities of the process $Y(k), k \geq 1$.

Proposition 2.1. The transition probabilities for the process $Y=\{Y(k), k \in \mathbb{N} \cup\{0\}\}$ have the following form:

$$
\begin{aligned}
& \operatorname{Pr}\{Y(2 k)=n \mid Y(2 k-1)=m\}=\left(\begin{array}{c}
2 m \\
n
\end{array}\right)\left(\frac{v(2 k)}{2 v(2 k-1)}\right)^{n}\left(1-\frac{v(2 k)}{2 v(2 k-1)}\right)^{2 m-n}, \quad n=0, \ldots, 2 m ; \\
& \operatorname{Pr}\{Y(2 k+1)=n \mid Y(2 k)=m\}= \begin{cases}1 & \text { if } n=2 m \\
0 & \text { otherwise. }\end{cases}
\end{aligned}
$$

Proof. Let $X_{i}(j-1)$ be a r.v. taking values 1 (if the $i$ th particle lying at level $j-1$ descends to an escape node at level $j$ ) and 0 (if the particle is captured by an absorbing state of level $j$ ). This means that $X_{i}(j-1)$ is a Bernoulli random variable with $\operatorname{Pr}\left\{X_{i}(j-1)=1\right\}=\rho(j-1)$. Note that the distribution of $X_{i}(j)$ is not affected by $i$. In the light of all this we can represent the number of particles at level $j$ by means of the random sum

$$
Y(j)=\sum_{i=1}^{2 Y(j-1)} X_{i}(j-1)
$$

The branching process, which implies the duplication of particles at each step, explains the $2 Y(j-1)$ appearing in $(2.13)$. The distribution (2.11) immediately follows from (2.13) while (2.12) is an immediate consequence of the definition of the process.

\section{Some results about the moments of $Y(k)$}

From the representation (2.13) we can immediately obtain some results by applying Wald's formulas. 
Theorem 3.1. We have the following recursive relationships

$$
\begin{aligned}
& \mathbb{E} Y(j)=2 \rho(j-1) \mathbb{E} Y(j-1)=\frac{v(j)}{v(j-1)} \mathbb{E} Y(j-1) \\
& \operatorname{Var} Y(j)=2 \rho(j-1)(1-\rho(j-1)) \mathbb{E} Y(j-1)+4 \rho^{2}(j-1) \operatorname{Var} Y(j-1) \\
& N_{Y(j)}(t)=\mathbb{E} t^{Y(j)}=N_{Y(j-1)}\left((1-\rho(j-1)+t \rho(j-1))^{2}\right) .
\end{aligned}
$$

Proof. Formula (3.3) can be obtained straightforwardly because

$$
N_{Y(j)}=\mathbb{E}\left(\mathbb{E} t^{X(j-1)}\right)^{2 Y(j-1)} .
$$

Formulas (3.1) and (3.2) are applications of Wald's formulas for random sums.

Remark 3.1. For odd values $j=2 k+1$, the formulas of Theorem 3.1 simplify as follows:

$$
\begin{aligned}
& \mathbb{E} Y(2 k+1)=2 \mathbb{E} Y(2 k) \\
& \operatorname{Var} Y(2 k+1)=4 \operatorname{Var} Y(2 k) \\
& N_{Y(2 k+1)}(t)=N_{Y(2 k)}\left(t^{2}\right)
\end{aligned}
$$

because $\rho(2 k)=v(2 k+1) / 2 v(2 k)=1$.

With some effort we can extract from Theorem 3.1 the explicit expressions of the mean values and variances of $Y(j)$.

Theorem 3.2. We have the following explicit values, for all $j \geq 1$

$$
\begin{aligned}
& \mathbb{E} Y(j)=v(j) \\
& \operatorname{Var} Y(2 k)=\frac{3}{4} v^{2}(2 k) \sum_{j=0}^{k} \frac{1}{v(j)}+\frac{v(2 k)}{4}-v^{2}(2 k)
\end{aligned}
$$

while $\operatorname{Var} Y(2 k+1)=4 \mathbb{V a r} Y(2 k)$ because of (3.5).

Proof. From (3.1), since $v(0)=1$ and $Y(0)=1$, we have immediately that

$$
\mathbb{E} Y(j)=\frac{v(j)}{v(j-1)} \cdot \frac{v(j-1)}{v(j-2)} \cdots \frac{v(1)}{v(0)} \mathbb{E} Y(0)=v(j) .
$$

For the proof of (3.8) we apply successively (3.5) and (3.2):

$$
\operatorname{Var} Y(2 k)=v(2 k)\left(1-\frac{v(2 k)}{2 v(2 k-1)}\right)+\frac{v^{2}(2 k)}{v^{2}(2 k-1)} \operatorname{Var} Y(2 k-1) .
$$

We then get that

$$
\begin{aligned}
\operatorname{Var}(Y(2 k))= & v(2 k)\left(1-\frac{v(2 k)}{2 v(2 k-1)}\right)+\frac{v^{2}(2 k)}{v^{2}(2 k-1)} \frac{v^{2}(2 k-1)}{v^{2}(2 k-2)} \operatorname{Var}(Y(2 k-2)) \\
= & v(2 k)-\frac{v^{2}(2 k)}{2 v(2 k-1)}+\frac{v^{2}(2 k)}{v^{2}(2 k-2)}\left[v(2 k-2)\left(1-\frac{v(2 k-2)}{2 v(2 k-3)}\right)\right. \\
& \left.+\frac{v^{2}(2 k-2)}{v^{2}(2 k-3)} \operatorname{Var}(Y(2 k-3))\right] \\
= & v(2 k)+\frac{v^{2}(2 k)}{v(2 k-2)}-\frac{v^{2}(2 k)}{2}\left(\frac{1}{v(2 k-1)}+\frac{1}{v(2 k-3)}\right) \\
& +\frac{v^{2}(2 k)}{v^{2}(2 k-3)} \frac{v^{2}(2 k-3)}{v^{2}(2 k-4)} \operatorname{Var}(Y(2 k-4))=\cdots \\
= & v(2 k)+v^{2}(2 k) \sum_{j=1}^{k-1} \frac{1}{v(2 k-2 j)}-\frac{v^{2}(2 k)}{2} \sum_{j=1}^{k} \frac{1}{v(2 k-2 j+1)}
\end{aligned}
$$


In order to explain the range of the sums appearing in the last member of (3.10) we note that the final application of (3.9) yields

$$
\begin{aligned}
\operatorname{Var}(Y(2 k)) & =v(2 k)+\sum_{j=1}^{k-2} \frac{v^{2}(2 k)}{v(2 k-2 j)}-\sum_{j=1}^{k-1} \frac{v^{2}(2 k)}{2 v(2 k-2 j+1)}+\frac{v^{2}(2 k)}{v^{2}(3)}\left[\frac{v^{2}(3)}{v^{2}(2)} \operatorname{Var}(Y(2))\right] \\
& =v(2 k)+\sum_{j=1}^{k-2} \frac{v^{2}(2 k)}{v(2 k-2 j)}-\sum_{j=1}^{k-1} \frac{v^{2}(2 k)}{2 v(2 k-2 j+1)}+\frac{v^{2}(2 k)}{v^{2}(2)}\left[v(2)\left(1-\frac{v(2)}{2 v(1)}\right)+\frac{v^{2}(2)}{v^{2}(1)} \operatorname{Var}(Y(1))\right] \\
& =v(2 k)+\sum_{j=1}^{k-2} \frac{v^{2}(2 k)}{v(2 k-2 j)}-\frac{1}{2} \sum_{j=1}^{k-1} \frac{v^{2}(2 k)}{v(2 k-2 j+1)}+\frac{v^{2}(2 k)}{v(2)}-\frac{v^{2}(2 k)}{2 v(1)} .
\end{aligned}
$$

In the last step the reader must take into account that $\operatorname{Pr}\{Y(1)=2\}=1$.

The result (3.10) can be adjusted in the following manner:

$$
\begin{aligned}
\operatorname{Var}(Y(2 k)) & =\frac{3}{4} v(2 k)+\frac{1}{4} v(2 k)+v^{2}(2 k) \sum_{j=1}^{k-1}\left(\frac{1}{v(2 k-2 j)}-\frac{1}{2 v(2 k-2 j+1)}\right)-\frac{v^{2}(2 k)}{2 v(1)} \\
& =\frac{3}{4} \sum_{j=0}^{k-1} \frac{v^{2}(2 k)}{v(2 k-2 j)}+\frac{1}{4} v(2 k)-\frac{v^{2}(2 k)}{4}=\frac{3}{4} \sum_{j=0}^{k-2} \frac{v^{2}(2 k)}{v(2 k-2 j)}+\frac{1}{4} v(2 k)+\frac{v^{2}(2 k)}{8}
\end{aligned}
$$

where we have used the fact that $2 v(2 k-2 j+1)=4 v(2 k-2 j)$. The final step consists in applying Lemma 2.1 and observing that:

$$
\sum_{j=0}^{k-2} \frac{1}{v(2 k-2 j)}=\sum_{h=0}^{k} \frac{1}{v(2 h)}-\frac{1}{v(0)}-\frac{1}{v(2)}=\sum_{h=0}^{k} \frac{1}{v(2 h)}-\frac{3}{2}=\sum_{h=0}^{k} \frac{1}{v(h)}-\frac{3}{2}
$$

so that

$$
\begin{aligned}
\operatorname{Var}(Y(2 k)) & =\frac{3}{4} v^{2}(2 k) \sum_{j=0}^{k} \frac{1}{v(j)}-\frac{3}{4} v^{2}(2 k) \frac{3}{2}+\frac{v^{2}(2 k)}{8}+\frac{v(2 k)}{4} \\
& =\frac{3}{4} v^{2}(2 k) \sum_{j=0}^{k} \frac{1}{v(j)}+\frac{v(2 k)}{4}-v^{2}(2 k)
\end{aligned}
$$

and this concludes the proof of (3.8).

Remark 3.2. For $k=2^{m-1}-1$ we can explicitly evaluate (3.11) because of (2.9) and keeping in mind that $v(2 k)=v(k)$ :

$$
\begin{aligned}
& \operatorname{Var}\left\{Y\left(2\left(2^{m-1}-1\right)\right)\right\}=\frac{3}{4} v^{2}\left(2^{m-1}-1\right)\left(\frac{3}{2}\right)^{m-1}-v^{2}\left(2^{m-1}-1\right)+\frac{v\left(2^{m-1}-1\right)}{4} \\
& \quad \text { by } \stackrel{(2.4)}{=} \frac{3}{4} 2^{2 m-2}\left(\frac{3}{2}\right)^{m-1}-2^{2 m-2}+\frac{2^{m-1}}{2^{2}}=3^{m} \cdot 2^{m-3}-2^{2 m-2}+2^{m-3} \\
& =2^{m-3}\left[3^{m}+1-2^{m+1}\right] .
\end{aligned}
$$

By similar calculations, since $v\left(2^{m-1}\right)=2$, we have that

$$
\begin{aligned}
\operatorname{Var}\left(Y\left(2^{m}\right)\right) & =\frac{3}{4} \cdot 2^{2}\left[\sum_{h=0}^{2^{m-1}-1} \frac{1}{v(h)}+\frac{1}{v\left(2^{m-1}\right)}\right]-2^{2}+\frac{1}{2} \\
& =\frac{3}{4} \cdot 2^{2}\left[\left(\frac{3}{2}\right)^{m-1}+\frac{1}{2}\right]+\frac{1}{2}-4=3^{m} \cdot 2^{m-1}-2 .
\end{aligned}
$$

Remark 3.3. If we write (3.3) as follows

$$
N_{Y(k)}(t)=N_{2 Y(k-1)}\left(1-\frac{v(k)}{2 v(k-1)}+\frac{v(k)}{2 v(k-1)} t\right)
$$


and then we derive (3.12) $j$ times with respect to $t$, we easily get the following relationship for the factorial moments:

$$
\mathbb{E}[Y(k) \cdots(Y(k)-j+1)]=\left(\frac{v(k)}{2 v(k-1)}\right)^{j} \mathbb{E}[2 Y(k)(2 Y(k)-1) \cdots(2 Y(k)-j+1)] .
$$

Remark 3.4. By applying (3.3) twice we get that

$$
\begin{aligned}
\mathbb{E} t^{Y(k)} & =\mathbb{E}\left[\left(1-\frac{v(k)}{2 v(k-1)}+\frac{v(k)}{2 v(k-1)} t\right)^{2}\right]^{Y(k-1)} \\
& =\mathbb{E}\left[1-\frac{v(k-1)}{2 v(k-2)}+\frac{v(k-1)}{2 v(k-2)}\left(1-\frac{v(k)}{2 v(k-1)}+\frac{v(k)}{2 v(k-1)} t\right)^{2}\right]^{2 Y(k-2)}
\end{aligned}
$$

If $k$ is even, then $v(k-1) / 2 v(k-2)=1$ and (3.13) becomes

$$
\mathbb{E} t^{Y(k)}=\mathbb{E}\left(1-\frac{v(k)}{2 v(k-1)}+\frac{v(k)}{2 v(k-1)} t\right)^{4 Y(k-2)}
$$

while for an odd $k$ we have that

$$
\mathbb{E} t^{Y(k)}=\mathbb{E} t^{2 Y(k-1)}=\mathbb{E}\left(1-\frac{v(k-1)}{2 v(k-2)}+\frac{v(k-1)}{2 v(k-2)} t^{2}\right)^{2 Y(k-2)}
$$

By applying successively the above relationships, one gets a cumbersome formula which cannot be further developed.

Although an exact expression for $\mathbb{E} t^{Y(k)}$ cannot be obtained for all $k$, we are able to provide a lower bound in the next theorem.

Theorem 3.3. For all $k \geq 1$ and $0<t \leq 1$,

$$
N_{Y(k)}(t)=\mathbb{E} t^{Y(k)} \geq\left(1-\frac{v(k)}{2^{k}}+\frac{v(k)}{2^{k}} t\right)^{2^{k}}=\mathbb{E} t^{\operatorname{Bin}\left(2^{k}, v(k) / 2^{k}\right)} .
$$

Proof. In view of Theorem 3.1 we have that

$$
\begin{aligned}
\mathbb{E} t^{Y(k)} & =\mathbb{E}\left(1-\frac{v(k)(1-t)}{2 v(k-1)}\right)^{2 Y(k-1)}=\mathbb{E}\left\{\mathbb{E}\left(\left(1-\frac{v(k)(1-t)}{2 v(k-1)}\right)^{2 Y(k-1)} \mid Y(k-2)\right)\right\} \\
& \geq \mathbb{E}\left\{\left(\mathbb{E}\left(1-\frac{v(k)}{2 v(k-1)}(1-t)\right)^{Y(k-1)} \mid Y(k-2)\right)^{2}\right\}
\end{aligned}
$$

where in the last step the conditional Jensen inequality

$$
\mathbb{E}\left[u^{2 Y(k-1)} \mid Y(k-2)\right] \geq\left[\mathbb{E} u^{Y(k-1)} \mid Y(k-2)\right]^{2}
$$

is applied.

By denoting $Z(k) \sim \operatorname{Bin}(\rho(k), 2 Y(k))$, the conditional mean in the last member of (3.17) can be easily evaluated as follows:

$$
\begin{aligned}
& \mathbb{E}\left\{\left(1-\frac{v(k)(1-t)}{2 v(k-1)}\right)^{Y(k-1)} \mid Y(k-2)\right\}=N_{Z(k-2)}\left(1-\frac{v(k)(1-t)}{2 v(k-1)}\right) \\
& =\left(1-\frac{v(k-1)}{2 v(k-2)}+\frac{v(k-1)}{2 v(k-2)}\left(1-\frac{v(k)}{2 v(k-1)}+\frac{v(k)}{2 v(k-1)} t\right)\right)^{2 Y(k-2)} \\
& =\left(1-\frac{v(k)}{2^{2} v(k-2)}+\frac{v(k)}{2^{2} v(k-2)} t\right)^{2 Y(k-2)} .
\end{aligned}
$$

By plugging (3.18) into (3.17) we get that 


$$
\begin{aligned}
\mathbb{E} t^{Y(k)} & \geq \mathbb{E}\left(1-\frac{v(k)(1-t)}{2^{2} v(k-2)}\right)^{2^{2} Y(k-2)}=\mathbb{E}\left(\mathbb{E}\left(1-\frac{v(k)(1-t)}{2^{2} v(k-2)}\right)^{2^{2} Y(k-2)} \mid Y(k-3)\right) \\
& \geq \mathbb{E}\left(\mathbb{E}\left(1-\frac{v(k)(1-t)}{2^{2} v(k-2)}\right)^{Y(k-2)} \mid Y(k-3)\right)^{2^{2}} \\
& =\mathbb{E}\left(1-\frac{v(k)(1-t)}{2^{3} v(k-3)}\right)^{2^{3} Y(k-3)} \geq \cdots \geq \mathbb{E}\left(1-\frac{v(k)(1-t)}{2^{k} v(0)}\right)^{2^{k} Y(0)}
\end{aligned}
$$

and this concludes the proof.

Remark 3.5. For $k=2^{m}, m \geq 1$, Theorems 3.1 and 3.3 permit us to write that

$$
\mathbb{E} t^{Y\left(2^{m}\right)}=\mathbb{E}\left(1-\frac{1}{2^{m}}+\frac{1}{2^{m}} t\right)^{2 Y\left(2^{m}-1\right)} \geq\left(1-\frac{2}{2^{2^{m}}}+\frac{2}{2^{2^{m}}} t\right)^{2^{2^{m}}} .
$$

We now have the following general result for conditional means.

Theorem 3.4. For every $0<j \leq k$ we have that

$$
\mathbb{E}\{Y(k) \mid Y(j)\}=\frac{v(k)}{v(j)} Y(j) .
$$

Proof. For all $k \geq j>0$, we clearly have that

$$
\begin{aligned}
\mathbb{E}\{Y(k) \mid Y(j)=h\} & =\sum_{l=0}^{2^{k-1}} \mathbb{E}\{Y(k) \mid Y(k-1)=l, Y(j)=h\} \operatorname{Pr}\{Y(k-1)=l \mid Y(j)=h\} \\
& =\sum_{l=0}^{2^{k-1}} \mathbb{E}\{Y(k) \mid Y(k-1)=l\} \operatorname{Pr}\{Y(k-1)=l \mid Y(j)=h\} \\
& =\sum_{l=0}^{2^{k-1}} 2 l \rho(k-1) \operatorname{Pr}\{Y(k-1)=l \mid Y(j)=h\}=2 \rho(k-1) \mathbb{E}\{Y(k-1) \mid Y(j)=h\}
\end{aligned}
$$

which easily implies the result:

$$
\mathbb{E}\{Y(k) \mid Y(j)=h\}=\frac{v(k)}{v(k-1)} \cdot \frac{v(k-1)}{v(k-2)} \cdots \frac{v(j+1)}{v(j)} \mathbb{E}\{Y(j) \mid Y(j)=h\}=\frac{v(k)}{v(j)} h .
$$

Corollary 3.1. We have that

$$
\mathbb{E}\left\{Y\left(r \cdot 2^{k}\right) \mid Y\left(r \cdot 2^{j}\right)\right\}=Y\left(r \cdot 2^{j}\right),
$$

for all $r \in \mathbb{N}$ and for all $k \geq j>0$. Thus the subsequences $Y\left(r \cdot 2^{k}\right), k \geq 0$ are martingales with respect to the natural filtrations (generated by $Y\left(r \cdot 2^{k}\right)$ ).

Q2 Proof. From Theorem $3.4 \mathbb{E}\left\{Y\left(r \cdot 2^{k}\right) \mid Y\left(r \cdot 2^{j}\right)\right\}=\frac{v\left(r \cdot 2^{k}\right)}{v\left(r \cdot 2^{j}\right)} Y\left(r \cdot 2^{j}\right)$ while from Lemma $2.1 v(r)=v(2 r)=\cdots=v\left(2^{j} r\right)=$ $\cdots=v\left(2^{k} r\right)$ and this proves the claim.

Remark 3.6. As a particular case, from Corollary 3.1 we derive that the subsequence $Y\left(2^{k}\right), k \geq 0$, which represents the number of particles reaching the base of the pregaskets $G_{k}$, is a martingale.

Remark 3.7. If we analyze the subsequence $Y\left(2^{k}\right), k \geq 0$, we can attack the problem in a different way. The process $Y\left(2^{k}\right), k \geq 0$ represents the number of particles on the outer nodes of the base of the pregasket $G_{k}$. If we denote by $W_{1}(k)$ and $W_{2}(k)$ the number of particles of the left-most and right-most nodes, respectively, then

$$
Y\left(2^{k}\right)=W_{1}\left(2^{k}\right)+W_{2}\left(2^{k}\right) .
$$

The r.v.'s $W_{j}\left(2^{k}\right), j=1,2$ form two branching processes which develop independently.

The processes $W_{j}(k), j=1,2$ have Bernoulli offspring distributions with mean one and therefore are critical (that is, extinguish with probability one). Then, also $Y\left(2^{k}\right)$ disappears with probability one. 


\section{Extinction probabilities}

We devote this section to the analysis of the probabilities of extinction which are a major feature of all branching processes.

We now study the probability of the following events:

$E_{k}=\{Y(k)=0\}=\{$ extinction occurred at or before generation (level) $k\}$.

We remark that the falling particles die if and only if they reach the absorbing nodes from which the further downward motion is not possible. This means that extinction of particles is strictly related to the dynamics inside the Sierpinski gasket.

From the relationship (3.3) we can infer that:

$$
\operatorname{Pr}\left\{E_{k}\right\}=N_{Y(k)}(0)=N_{Y(k-1)}\left((1-\rho(k-1))^{2}\right)=\mathbb{E}\left(1-\frac{v(k)}{2 v(k-1)}\right)^{2 Y(k-1)} .
$$

In view of the autosimilarity of the pregasket $G_{m}$, we have the following recursive relationship for the probability of extinction of the subsequence $Y\left(2^{m}\right), m \geq 0$. Loosely speaking, the autosimilarity consists in that the pregasket $G_{m-1}$ is îdentical to a rescaled version of the three biggest triangles forming the pregasket $G_{m}$.

Theorem 4.1. The following recursive relationship holds

$$
\operatorname{Pr}\left\{E_{2^{m}}\right\}=\mathbb{E}\left(1-\frac{1-\operatorname{Pr}\left\{E_{2^{m-1}}\right\}}{2^{m-1}}\right)^{2 Y\left(2^{m-1}-1\right)} .
$$

\section{Proof.}

$$
\operatorname{Pr}\left\{E_{2^{m}}\right\}=\operatorname{Pr}\left\{Y\left(2^{m}\right)=0\right\}=\sum_{h=0}^{2^{2^{m-1}}} \operatorname{Pr}\left\{Y\left(2^{m}\right)=0 \mid Y\left(2^{m-1}\right)=h\right\} \operatorname{Pr}\left\{Y\left(2^{m-1}\right)=h\right\} .
$$

By autosimilarity, the rules governing the branching process are the same for all the pregaskets $G_{m-1}$ composing $G_{m}$, for all $m \geq 1$. In other words, the subprocess $\left\{Y\left(2^{m}\right), m \geq 0\right\}$ is stationary, that is $\operatorname{Pr}\left\{Y\left(2^{m}\right)=0 \mid Y\left(2^{m-1}\right)=h\right\}=$ $\operatorname{Pr}\left\{Y\left(2^{m-1}\right)=0 \mid Y(0)=h\right\}$. Thus, in view of the independence of particles,

$$
\begin{aligned}
\operatorname{Pr}\left\{E_{2^{m}}\right\} & =\sum_{h=0}^{2^{2^{m-1}}} \operatorname{Pr}\left\{Y\left(2^{m-1}\right)=0 \mid Y(0)=h\right\} \operatorname{Pr}\left\{Y\left(2^{m-1}\right)=h\right\} \\
& =\sum_{h=0}^{2^{2^{m-1}}}\left[\operatorname{Pr}\left\{Y\left(2^{m-1}\right)=0\right\}\right]^{h} \operatorname{Pr}\left\{Y\left(2^{m-1}\right)=h\right\} \\
& =N_{Y\left(2^{m-1}\right)}\left(\operatorname{Pr}\left\{E_{2^{m-1}}\right\}\right)=N_{Y\left(2^{m-1}-1\right)}\left(1-\rho\left(2^{m-1}-1\right)\left(1-\operatorname{Pr}\left\{E_{2^{m-1}}\right\}\right)\right) .
\end{aligned}
$$

Formula (4.3) emerges once the probability $\rho\left(2^{m-1}-1\right)=1 / 2^{m-1}$ is taken into account.

Remark 4.1. From Theorem 3.3 for $t=0$ we get that

$$
\operatorname{Pr}\left\{E_{k}\right\}=\left.\mathbb{E} t^{Y(k)}\right|_{t=0} \geq\left(1-\frac{\nu(k)}{2^{k}}\right)^{2^{k}} .
$$

From (4.2) and the Jensen inequality, we have instead the lower bound:

$$
\operatorname{Pr}\left\{E_{k}\right\}=\mathbb{E}\left(1-\frac{v(k)}{2 v(k-1)}\right)^{2 Y(k-1)} \geq\left(1-\frac{v(k)}{2 v(k-1)}\right)^{2 \mathbb{E Y}(k-1)}=\left(1-\frac{v(k)}{2 v(k-1)}\right)^{2 v(k-1)} .
$$

In the intermediate steps we have applied the Jensen inequality to the convex function $g(x)=\left(1-\frac{v(k)}{2 v(k-1)}\right)^{2 x}$.

In view of the fact that $v(k-1) \leq 2^{k-1}$ and that the function $g(x)=(1-a / x)^{x}$ is increasing, the lower bound (4.4) is stricter than (4.5).

A sequence of lower bounds for $\left\{Y\left(2^{m}\right), m \geq 0\right\}$, can be written down by applying (4.3) recursively together with the Jensen inequality. For this purpose we consider the sequence defined by

$$
l_{m}=\left(1-\frac{1-l_{m-1}}{2^{m-1}}\right)^{2^{m}}, \quad m \geq 2
$$


where $l_{1}=\operatorname{Pr}\left\{E_{2}\right\}=1 / 16$. In order to show that $l_{m} \leq \operatorname{Pr}\left\{E_{2^{m}}\right\}$, we examine some special cases which illustrate the procedure. For $m=2$,

$$
\operatorname{Pr}\left\{E_{2^{2}}\right\}=\mathbb{E}\left(1-\frac{1-\operatorname{Pr}\left\{E_{2}\right\}}{2}\right)^{2 Y(1)} \geq\left(1-\frac{1}{2}\left(1-l_{1}\right)\right)^{2 \mathbb{E} Y(1)}=\left(1-\frac{1}{2}\left(1-l_{1}\right)\right)^{2^{2}}=l_{2} .
$$

By similar steps we have that

$$
\operatorname{Pr}\left\{E_{2^{3}}\right\}=\mathbb{E}\left(1-\frac{1-\operatorname{Pr}\left\{E_{2^{2}}\right\}}{2^{2}}\right)^{2 Y(2)} \geq\left(1-\frac{\left(1-l_{2}\right)}{2^{2}}\right)^{2 \mathbb{E} Y(2)}=\left(1-\frac{\left(1-l_{2}\right)}{2^{2}}\right)^{2^{3}}=l_{3} .
$$

By iterating this procedure we arrive at $\operatorname{Pr}\left\{E_{2^{m}}\right\} \geq l_{m}$.

It is easily seen that the sequence $l_{m}$ is bounded and increasing. Then, by passing to the limit, we get that

$$
l=\lim _{m \rightarrow \infty} l_{m}=\lim _{m \rightarrow \infty}\left(1-\frac{1-l_{m-1}}{2^{m-1}}\right)^{2^{m}}=\mathrm{e}^{-2 \lim _{m}\left(1-l_{m}\right)}=\mathrm{e}^{-2(1-l)} .
$$

That is, $l=\lim _{m} l_{m}$ is the solution to the transcendental equation

$$
l=\mathrm{e}^{-2(1-l)} \text {. }
$$

The root of Eq. (4.7) can be computed numerically and we get that $l^{*} \approx 0.203$. It is to worthwhile noting that $l_{m}$ is a significant lower bound for finite values of $m$ but not for $m \rightarrow \infty$, because from Remark 3.7 we know that $\operatorname{Pr}\left\{\lim _{m \rightarrow \infty} Y\left(2^{m}\right)=0\right\}=1$.

\section{Uncited references}

Procaccia and O'Shaughnessy, 1985.

\section{Acknowledgements}

We want to express our gratitude to Prof. Aimé Lachal for his comments and suggestions on a previous draft of this paper. Thanks are also due to the attentive referee who read and reported on this paper.

\section{References}

Barlow, M.T., 1998. Diffusions on fractals. In: Lectures on Probability Theory and Statistics, Saint-Flour, 1995. In: Lecture Notes in Math., vol. 1690. Springer, Berlin, pp. 1-121.

Barlow, M.T., Bass, R.F., 1989. The construction of Brownian motion on the Sierpinski carpet. Ann. Inst. Henri Poincaré 25 (3), $225-257$.

Barlow, M.T., Perkins, E.A., 1988. Brownian motion on the Sierpinski gasket. Probab. Theory Related Fields 79, 543-623.

Bramson, M.D., 1978. Maximal displacement of branching Brownian motion. Comm. Pure Appl. Math. 31 (5), 531-581.

Dafydd Jones, O., 1996. Transition probabilities for the simple random walk on the Sierpinski graph. Stoch. Process. Appl. 61, 45-69.

Kumagai, T., 1993. Estimates of the transition densities for Brownian motion on nested fractals. Probab. Theory Related Fields 96, $205-224$.

Leorato, S., Orsingher, E., 2007. A grain of dust falling through a Sierpinski gasket. Acta Math. Sin. 23 (6), 1095-1108.

McKean, H.P., 1975. Application of Brownian motion to the equation of Kolmogorov-Petrovskii-Piskunov. Comm. Pure Appl. Math. 28 (3), $323-331$.

Metz, V., 1993. How many diffusions exist on the Vicsek snowflake? Acta Appl. Math. 32, 224-241.

Osada, H., 2007. Exotic Brownian motions. Kyushu J. Math. 61 (1), 233-257.

Procaccia, I., O'Shaughnessy, B., 1985. Diffusions on fractals. Phys. Rev. A 32 (5), 3073-3083.

Ratanov, N., 2006. Branching random motions, nonlinear hyperbolic systems and travelling waves. ESAIM Probab. Stat. 10, $236-257$.

Teufl, E., 2003. The average displacement of the simple random walk on the Sierpinski graph. Comb. Probab. Comput. 12, 203-222. 